\title{
Risk of lung cancer in patients with gastro-esophageal reflux disease: a population-based cohort study
}

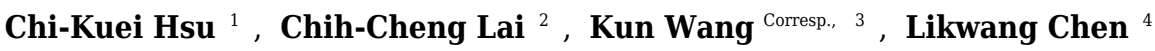 \\ ${ }^{1}$ Department of Internal Medicine, E-Da Hospital, Kaohsiung, Taiwan \\ 2 Department of Intensive Care Medicine, Chi Mei Medical Center, Liouying, Tainan, Taiwan \\ 3 Department of Internal Medicine, Cardinal Tien Hospital, New Taipei city, Taiwan \\ 4 National Health Research Institutes, Miaoli, Taiwan \\ Corresponding Author: Kun Wang \\ Email address: kunwang@mospital.com
}

This large-scale, controlled cohort study estimated the risks of lung cancer in patients with gastro-esophageal reflux disease (GERD) in Taiwan. We conducted this population-based study using data from the National Health Insurance Research Database of Taiwan during the period from 1997 to 2010. Patients with GERD were diagnosed using endoscopy, and controls were matched to patients with GERD at a ratio of 1:4. We identified 15,412 patients with GERD and 60,957 controls. Compared with the controls, the patients with GERD had higher rates of osteoporosis, diabetes mellitus, asthma, chronic obstructive pulmonary disease, pneumonia, bronchiectasis, depression, anxiety, hypertension, dyslipidemia, chronic liver disease, congestive heart failure, atrial fibrillation, stroke, chronic kidney disease, and coronary artery disease (all $P<.05$ ). A total of 85 patients had lung cancer among patients with GERD during the follow-up of 42,555 person-years, and the rate of lung cancer was 0.0020 per person-year. By contrast, 232 patients had lung cancer among patients without GERD during the follow-up of 175,319 person-years, and the rate of lung cancer was 0.0013 per person-year. By using stepwise Cox regression model, the overall incidence of lung cancer remained significantly higher in the patients with GERD than in the controls (hazard ratio, 1.53; $95 \% \mathrm{Cl}, 1.19-1.98$ ). The cumulative incidence of lung cancer was higher in the patients with GERD than in the controls ( $P=$ .0012). In conclusion, our large population-based cohort study provides evidence that GERD may increase the risk of lung cancer in Asians. 
1 Risk of lung cancer in patients with gastro-esophageal reflux disease: a

2 population-based cohort study

3 Running head: Lung cancer and GERD

4

5 Chi-Kuei Hsu ${ }^{1}$, Chih-Cheng Lai ${ }^{2}$, Kun Wang ${ }^{3 *}$, Likwang Chen ${ }^{4 *}$

$6 \quad{ }^{1}$ Department of Internal Medicine, E-Da Hospital, Kaohsiung, Taiwan

$7 \quad{ }^{2}$ Department of Intensive Care Medicine, Chi Mei Medical Center, Liouying, Tainan

$8 \quad{ }^{3}$ Department of Internal Medicine, Cardinal Tien Hospital, Fu Jen Catholic University College

9 of Medicine, New Taipei City

$10{ }^{4}$ Institute of Population Health Sciences, National Health Research Institutes, Zhunan, Miaoli

11 County, Taiwan

12 All authors have read and approved the manuscript.

13 Wang $\mathrm{K}$, and Chen LK contributed equally to this work.

14 *Correspondence to:

15 Dr. Kung Wang (E-mail: kunwang@mospital.com)

16 PhD. Likwang Chen (E-mail: likwang@nhri.org.tw) 


\section{ABSTRACT}

18 This large-scale, controlled cohort study estimated the risks of lung cancer in patients with 19 gastro-esophageal reflux disease (GERD) in Taiwan. We conducted this population-based study

20 using data from the National Health Insurance Research Database of Taiwan during the period

21 from 1997 to 2010. Patients with GERD were diagnosed using endoscopy, and controls were

22 matched to patients with GERD at a ratio of 1:4. We identified 15,412 patients with GERD and

2360,957 controls. Compared with the controls, the patients with GERD had higher rates of

24 osteoporosis, diabetes mellitus, asthma, chronic obstructive pulmonary disease, pneumonia,

25 bronchiectasis, depression, anxiety, hypertension, dyslipidemia, chronic liver disease, congestive

26 heart failure, atrial fibrillation, stroke, chronic kidney disease, and coronary artery disease (all P

$27<.05)$. A total of 85 patients had lung cancer among patients with GERD during the follow-up of

2842,555 person-years, and the rate of lung cancer was 0.0020 per person-year. By contrast, 232

29 patients had lung cancer among patients without GERD during the follow-up of 175,319 person-

30 years, and the rate of lung cancer was 0.0013 per person-year. By using stepwise Cox regression

31 model, the overall incidence of lung cancer remained significantly higher in the patients with

32 GERD than in the controls (hazard ratio, 1.53; 95\%CI, 1.19-1.98). The cumulative incidence of

33 lung cancer was higher in the patients with GERD than in the controls $(\mathrm{P}=.0012)$. In conclusion,

34 our large population-based cohort study provides evidence that GERD may increase the risk of

35 lung cancer in Asians. 


\section{INTRODUCTION}

39 Gastro-esophageal reflux disease (GERD) is a condition that develops when the reflux of

40 stomach contents causes troublesome symptoms and complications (Bredenoord, Pandolfino, \&

41 Smout, 2013; Moayyedi \& Talley, 2006; Vakil, van Zanten, Kahrilas, Dent, \& Jones, 2006).

42 GERD is a global issue that affects both children and adults (El-Serag, Sweet, Winchester, \&

43 Dent, 2014). The incidence of the disease appears to have increased during the past 2 decades,

44 particularly in North America and East Asia (El-Serag, 2007; El-Serag et al., 2014; Vakil, 2010).

45 The most common manifestations of GERD are esophageal symptoms, including heartburn,

46 dysphagia, and regurgitation, and it can cause extra-esophageal presentation such as

47 bronchospasm, laryngitis, and chronic cough. Because it may cause lung injury from recurrent

48 microaspiration, GERD is associated with the risk of several lung diseases, such as idiopathic

49 pulmonary fibrosis, cystic fibrosis, connective tissue disease, asthma, chronic obstructive

50 pulmonary disease (COPD), and interstitial lung disease (Blondeau et al., 2008; D'Ovidio et al.,

51 2005; Mise et al., 2010; Morehead, 2009; Pacheco-Galvan, Hart, \& Morice, 2011; Pashinsky,

52 Jaffin, \& Litle, 2009; Salvioli et al., 2006; Sweet, Patti, Hoopes, Hays, \& Golden, 2009).

53 Recently, Vereczkei et al (Vereczkei, Horvath, Varga, \& Molnar, 2008) investigated the

54 association between GERD and non-small cell lung cancer (NSCLC) and found that a 55 considerably higher proportion of patients with NSCLC had GERD than the general population, 56 irrespective of cell type. Therefore, a study proposed that GERD-associated chronic lung injury

57 may be one element of lung cancer promotion (Herbella, Neto, Santoro, \& Figueiredo, 2015).

58 However, it enrolled only 25 patients with surgically treated adenocarcinoma and squamous cell

59 carcinoma, and the relationship between GERD and lung cancer remains unclear (Herbella et al.,

60 2015; Vereczkei et al., 2008). Therefore, whether GERD is associated with an increased risk of 
61 lung cancer should be determined. Hence, we performed a large-scale, controlled cohort study to

62 estimate the hazard rates of lung cancer in patients with GERD by using a nationwide, 63 population-based database in Taiwan. 


\section{MATERIALS \& METHODS}

\section{Data Source} that covers outpatient visits, hospital admissions, prescriptions, interventional procedures, and disease profiles for $>99 \%$ of the population of Taiwan (23.12 million people in 2009) (Chen, Muo, Lee, Yu, \& Sung, 2011). The Taiwan's National Health Research Institute (NHRI) used the original data from the NHI program to construct a longitudinal database of patients admitted between 1997 and 2010. This cohort includes 2,619,534 hospitalized patients, representing 10\% of all NHI enrollees. This sampled fraction (a 3.4:1 ratio) is based on a regulation that limits the maximal amount of NHI data that can be extracted for research purposes. The National Health Insurance Research Database (NHIRD) is one of the largest and most comprehensive databases worldwide and has been used extensively in various studies of prescription use, diagnoses, and hospitalizations. This study has obtained the approval of Institutional Review Board of Cardinal Tien Hospital (Number: EC1011008-E-R1).

\section{Identification of patients with GERD and without GERD}

To investigate the associations between GERD and the risk of lung cancer, we performed a

81 cohort study. All beneficiaries with GERD from 1997 to 2010 were extracted using the

82 International Classification of Diseases, Ninth Revision, Clinical Modification (ICD-9-CM)

83 codes 530.85, 530.11, and 530.81. Patients with GERD were identified using the ICD-9-CM 
84 codes and procedure codes for endoscopy as previous study (Lee et al, 2014). Patients who were

85 not diagnosed with GERD after receiving endoscopy were excluded. Patients with a history of

86 lung cancer or peptic ulcer disease were also excluded. We matched controls (patients without

87 GERD) to patients with GERD by age, sex, and the index date at a ratio of 1:4. In the non-GERD

88 group, patients with a history of lung cancer or peptic ulcer disease were excluded.

89 Baseline variables

90 We collected data on demographic and clinical characteristics of the study population,

91 including age, sex, and comorbidities. Comorbidities were defined according to the ICD-9-CM

92 and procedure codes within 1 year before index admission. We used a relatively strict criterion to

93 define comorbidities: coding one morbidity required at least one admission or 3 outpatient clinic

94 visits for disease treatment during the year before index admission.

\section{Definition of outcome}

96 We followed up each patient until December 31, 2010, to observe for the development of de

97 novo lung cancer. In Taiwan, patients with cancer can apply for a catastrophic illness certificate

98 that exempts them from any out-of-pocket expenses for cancer evaluation and care. The

99 development of de novo lung cancer was identified by ICD-9-CM code 162 having been noted

100 on the catastrophic illness certificate as previous study (Jian et al, 2015). The follow-up duration

101 was calculated from the date of GERD diagnosis (index date) to the date of the first recorded

102 cancer code. 


\section{Statistical analysis}

104 All data were analyzed using SAS Version 9.3 software (SAS Institute). Categorical variables

105 are expressed as numbers or percentages and were compared using the chi-square test. Incidence

106 rates of lung cancer in both GERD and non-GERD groups were calculated by Poisson regression.

107 The Kaplan-Meier method was used to estimate the cumulative incidence rate of lung cancer in

108 patients with or without GERD. The cumulative incidence curves of both groups were compared

109 using the log-rank test. We used Log-Minus-Log survival plots to evaluate proportional hazard

110 assumption. To assess the risk of lung cancer, a list of potential risk factors associated both with

111 admission of lung cancer and with GERD status was considered in the Cox regression model.

112 Univariable and multivariable Cox regression models (stepwise selection) were performed to

113 examine the association of lung cancer with potential confounding factors such as osteoporosis,

114 diabetes mellitus (DM), asthma, COPD, pneumonia, anxiety, hypertension, dyslipidemia, chronic

115 liver disease, congestive heart failure (CHF), atrial fibrillation, stroke, chronic kidney disease

116 (CKD), and coronary artery disease (CAD). Two-sided $\mathrm{P}$ values $<.05$ were considered

117 statistically significant. 


\section{RESULTS}

119 Initially, the NHIRD was used to identify 97,221 patients diagnosed with GERD after

120 undergoing esophagogastroduodenoscopy from January 1, 1997 to December 31, 2010. After

121 excluding 943 patients aged $<18$ or $>100$ years, 71,255 patients diagnosed with lung cancer or

122 peptic ulcer before the index date, 9,397 patients without GERD diagnosis 1 year later after the

123 index date, and 182 patients with missing demographic data, we found only 15,444 patients

124 eligible for matching. Overall, we identified 15,444 patients with GERD and 60,957 age- and

125 sex-matched controls (Figure 1).

126 A total of 85 patients had lung cancer among patients with GERD during the follow-up of 42,555

127 person-years, and the rate of lung cancer was 0.0020 per person-year. By contrast, 232 patients

128 without GERD had lung cancer during the follow-up of 175,319 person-years, and the rate of

129 lung cancer was 0.0013 per person-year (Table 1). The baseline characteristics and comorbidities

130 are listed in Table 2. Compared with the controls, the patients with GERD displayed higher rates

131 of osteoporosis, asthma, COPD, pneumonia, bronchiectasis, depression, anxiety, hypertension,

132 dyslipidemia, chronic liver disease, $\mathrm{CHF}$, atrial fibrillation, stroke, CKD, and CAD (all P $<.05)$.

133 There is no violation of proportional hazard assumption. By using stepwise Cox regression

134 model, we found all potential confounding variables are not significant associated with lung 135 cancer except for GERD (HR, 1.53; 95\%CI, 1.19-1.98; Table 3). As shown in Figure 2, the 136 cumulative incidence of lung cancer was higher in the patients with GERD than in the controls $137(\mathrm{P}=.0012)$. 


\section{DISCUSSION}

139 This large, population-based, long-term follow-up cohort study is the first to investigate the

140 relationship between GERD and lung cancer. Besides the strong association between GERD and

141 esophageal cancer, several studies have shown that GERD is also an important risk factor for

142 laryngeal/pharyngeal cancer (Bacciu et al., 2004; Langevin et al., 2013; Vaezi, Qadeer, Lopez, \&

143 Colabianchi, 2006). Additionally, the significant association between GERD and laryngeal

144 cancerwith pooled odds ratios of 2.86 (95\% CI, 2.73-2.99) and 2.37 (95\% CI 1.38-4.08) on the

145 basis of fixed-effect and random-effect models, respectively, were demonstrated in one meta-

146 analysis (Qadeer, Colabianchi, \& Vaezi, 2005). Although it may be logical that the lungs as one

147 of organ near esophagus, and supposed to be affected by the gastric refluxate, no study has

148 assessed the possible relationship between GERD and lung cancer. Our study is the first to

149 demonstrate a significant positive association between GERD and lung cancer. This finding was

150 supported by the increased risk of lung cancer in comparison with age- and sex-matched controls

151 (crude HR, 1.53; 95\%CI, 1.19-1.98). Our findings have some clinical implications. After

152 confirming this significant association between GERD and lung cancer, it was suggested that

153 aggressive treatment of GERD possibly preventing the development of lung cancer. However,

154 further studies should be warranted to prove the possible chemopreventive role of antacid use in

155 patients with GERD. 
156 Our study has several strengths. First, all of the patients with GERD and controls in this

157 study were enrolled from the Taiwan NHIRD, which is a highly representative database.

158 Therefore, the bias of recall and selection can be minimizing. Second, our study identified lung

159 cancer patients by using valid and definite approaches. In the Taiwan NHI program, individuals

160 with registration of cancer for a catastrophic illness certificate required biopsy and histological

161 verification. Third, by using medical records from NHIRD, we can reduce the likelihood of non-

162 response and loss of follow-up to mininum. Besides, there were some variables during the

163 multivariable analysis. We have controlled them by statistic methods (table 3). Most important of

164 all, we used a nationwide and population-based database - Taiwan NHIRD. Thus, the findings in

165 the present work can be generalized in the real world.

166 Several mechanisms can help explain the significant relationship between GERD and lung

167 cancer. First, several studies have shown that the refluxate can destroy the epithelium of the

168 larynx or pharynx by means of introducing chronic inflammation (Rees et al., 2008), or

169 activating proliferative signaling pathways (Dvorak et al., 2011; Johnston et al., 2012; Sung et al.,

170 2003), and further result in malignant transformation. In addition, based on the studies

171 investigating the pathogenesis of Barrett's esophagus and esophageal carcinoma, both acid and

172 bile can promate carcinogenesis through the induction of DNA damage and the influence of cell

173 proliferation and apoptosis (Denlinger \& Thompson, 2012; Fang et al., 2013). These 
174 pathogenesis may happen in the respiratory tract, and contribute to the development of lung

175 cancer. Second, the trend of the predominance of lung adenocarcinoma among all cell type is

176 similar with the distribution trend of esophageal cancer (Etzel et al., 2006; Liam, Pang, Leow,

177 Poosparajah, \& Menon, 2006). Third, the origin of central lung adenocarcinoma is different from

178 the peripheral lung cancer. Lung cancer at central site is more prone to be affected by gastric

179 refluxate than at a peripheral site. Thus, lung adenocarcinoma at central site is more likely to

180 arise in the glandular epithelium in contrast to lung cancer at a peripheral site which possibly

181 originates from type II pneumocytes and Clara cells (Fukui et al., 2013).

182 However, this study also had several limitations. First, we cannot obtain the data such as

183 smoking, which is an important risk factor for both GERD and lung cancer. However, we try to

184 include some smoking-related disorders such as dyslipidemia, hypertension, CAD, or COPD to

185 minimize the influence of smoking. In addition, the data regarding the type of lung cancer was

186 not available. Therefore, we cannot further analysis the association between GERD and the

187 specific type of lung cancer. Second, patients with GERD may more often visit physicians than

188 patients without GERD and this difference may cause possible surveillance bias. Finally, we did

189 not collect the data about the use of anti-GERD treatments such as proton pump inhibitors or

190 histamine-2-receptor antagonist.

\section{CONCLUSIONS}


192 Our large, population-based cohort study provides evidence that GERD may increase the risk of

193 lung cancer.

194

\section{Data Availability}

196 The following information was supplied regarding the deposition of related data: Raw data for

197 this work was obtained by application from the National Health Insurance Research Database,

198 Taiwan (http://nhird.nhri.org.tw/en/index.htm) and may not be shared according to the

199 Database's rules governing use. Access to the data used in this study may be obtained by citizens

200 of the Republic of China who fulfill the requirements of conducting research projects 
202

203

204

205

206

207

208

209

210

211

212

213

214

215

216

217

218

219

220

221

222

223

224

225

226

227

228

229

230

231

232

233

234

235

\section{REFERENCES}

Bacciu, A., Mercante, G., Ingegnoli, A., Ferri, T., Muzzetto, P., Leandro, G., . . . Bacciu, S. (2004). Effects of gastroesophageal reflux disease in laryngeal carcinoma. Clin Otolaryngol Allied Sci, 29(5), 545-548. doi: 10.1111/j.1365-2273.2004.00851.x

Blondeau, K., Dupont, L. J., Mertens, V., Verleden, G., Malfroot, A., Vandenplas, Y., . . Sifrim, D. (2008). Gastro-oesophageal reflux and aspiration of gastric contents in adult patients with cystic fibrosis. Gut, 57(8), 1049-1055. doi: 10.1136/gut.2007.146134

Bredenoord, A. J., Pandolfino, J. E., \& Smout, A. J. (2013). Gastro-oesophageal reflux disease. Lancet, 381(9881), 1933-1942. doi: 10.1016/s0140-6736(12)62171-0

Chen, P. C., Muo, C. H., Lee, Y. T., Yu, Y. H., \& Sung, F. C. (2011). Lung cancer and incidence of stroke: a population-based cohort study. Stroke, 42(11), 3034-3039. doi: 10.1161/strokeaha.111.615534

Denlinger, C. E., \& Thompson, R. K. (2012). Molecular basis of esophageal cancer development and progression. Surg Clin North Am, 92(5), 1089-1103. doi: 10.1016/j.suc.2012.07.002

D'Ovidio, F., Singer, L. G., Hadjiliadis, D., Pierre, A., Waddell, T. K., de Perrot, M., . . . Keshavjee, S. (2005). Prevalence of gastroesophageal reflux in end-stage lung disease candidates for lung transplant. Ann Thorac Surg, 80(4), 1254-1260. doi: 10.1016/j.athoracsur.2005.03.106

Dvorak, K., Goldman, A., Kong, J., Lynch, J. P., Hutchinson, L., Houghton, J. M., . . Westra, W. M. (2011). Molecular mechanisms of Barrett's esophagus and adenocarcinoma. Ann N Y Acad Sci, 1232, 381-391. doi: 10.1111/j.1749-6632.2011.06062.x

El-Serag, H. B. (2007). Time trends of gastroesophageal reflux disease: a systematic review. Clin Gastroenterol Hepatol, 5(1), 17-26. doi: 10.1016/j.cgh.2006.09.016

El-Serag, H. B., Sweet, S., Winchester, C. C., \& Dent, J. (2014). Update on the epidemiology of gastro-oesophageal reflux disease: a systematic review. Gut, 63(6), 871-880. doi: 10.1136/gutjnl-2012-304269

Etzel, C. J., Lu, M., Merriman, K., Liu, M., Vaporciyan, A., \& Spitz, M. R. (2006). An epidemiologic study of early onset lung cancer. Lung Cancer, 52(2), 129-134. doi: 10.1016/j.lungcan.2005.11.018

Fang, Y., Chen, X., Bajpai, M., Verma, A., Das, K. M., Souza, R. F., . . Dvorak, K. (2013). Cellular origins and molecular mechanisms of Barrett's esophagus and esophageal adenocarcinoma. Ann N Y Acad Sci, 1300, 187-199. doi: 10.1111/nyas.12249

Fukui, T., Shaykhiev, R., Agosto-Perez, F., Mezey, J. G., Downey, R. J., Travis, W. D., \& Crystal, R. G. (2013). Lung adenocarcinoma subtypes based on expression of human 
236

237

238

239

240

241

242

243

244

245

246

247

248

249

250

251

252

253

254

255

256

257

258

259

260

261

262

263

264

265

266

267

268

269

270

airway basal cell genes. Eur Respir $J, \quad 42(5), \quad 1332-1344 . \quad$ doi: 10.1183/09031936.00144012

Herbella, F. A., Neto, S. P., Santoro, I. L., \& Figueiredo, L. C. (2015). Gastroesophageal reflux disease and non-esophageal cancer. World J Gastroenterol, 21(3), 815-819. doi: 10.3748/wjg.v21.i3.815

Jian, Z. H., Huang, J. Y., Ko, P. C., Jan, S.. R., Nfor, O. N., Lung, C. C., Ku, W. Y., Ho, C. C., Pan, H. H., \& Liaw, Y. P. (2015). Impact of coexisting pulmonary diseases on survival of patients with lung adenocarcinoma: a STROBE-compliant article. Medicine (Baltimore). 94(4):e443. doi: 10.1097/MD.0000000000000443.

Johnston, N., Yan, J. C., Hoekzema, C. R., Samuels, T. L., Stoner, G. D., Blumin, J. H., \& Bock, J. M. (2012). Pepsin promotes proliferation of laryngeal and pharyngeal epithelial cells. Laryngoscope, 122(6), 1317-1325. doi: 10.1002/lary.23307

Langevin, S. M., Michaud, D. S., Marsit, C. J., Nelson, H. H., Birnbaum, A. E., Eliot, M., . . Kelsey, K. T. (2013). Gastric reflux is an independent risk factor for laryngopharyngeal carcinoma. Cancer Epidemiol Biomarkers Prev, 22(6), 1061-1068. doi: 10.1158/10559965.epi-13-0183

Liam, C. K., Pang, Y. K., Leow, C. H., Poosparajah, S., \& Menon, A. (2006). Changes in the distribution of lung cancer cell types and patient demography in a developing multiracial Asian country: experience of a university teaching hospital. Lung Cancer, 53(1), 23-30. doi: 10.1016/j.lungcan.2006.03.009

Lee, Y. L., Hu, H. Y., Yang, N. P., Chou, P., \& Chu D. (2014). Dental prophylaxis decreases the risk of esophageal cancer in males; a nationwide population-based study in Taiwan. PLoS One 9(10):e109444. doi: 10.1371/journal.pone.0109444. eCollection 2014.

Mise, K., Capkun, V., Jurcev-Savicevic, A., Sundov, Z., Bradaric, A., \& Mladinov, S. (2010). The influence of gastroesophageal reflux in the lung: a case-control study. Respirology, 15(5), 837-842. doi: 10.1111/j.1440-1843.2010.01777.x

Moayyedi, P., \& Talley, N. J. (2006). Gastro-oesophageal reflux disease. Lancet, 367(9528), 2086-2100. doi: 10.1016/s0140-6736(06)68932-0

Morehead, R. S. (2009). Gastro-oesophageal reflux disease and non-asthma lung disease. Eur Respir Rev, 18(114), 233-243. doi: 10.1183/09059180.00002509

Pacheco-Galvan, A., Hart, S. P., \& Morice, A. H. (2011). Relationship between gastrooesophageal reflux and airway diseases: the airway reflux paradigm. Arch Bronconeumol, 47(4), 195-203. doi: 10.1016/j.arbres.2011.02.001

Pashinsky, Y. Y., Jaffin, B. W., \& Litle, V. R. (2009). Gastroesophageal reflux disease and idiopathic pulmonary fibrosis. Mt Sinai J Med, 76(1), 24-29. doi: 10.1002/msj.20088 
271 Patti, M. G., Gasper, W. J., Fisichella, P. M., Nipomnick, I., \& Palazzo, F. (2008).

272

273

274

275

276

277

278

279

280

281

282

283

284

285

286

287

288

289

290

291

292

293

294

295

296

297

298

299

300

301

302

303 Gastroesophageal reflux disease and connective tissue disorders: pathophysiology and implications for treatment. J Gastrointest Surg, 12(11), 1900-1906. doi: 10.1007/s11605008-0674-9

Qadeer, M. A., Colabianchi, N., \& Vaezi, M. F. (2005). Is GERD a risk factor for laryngeal cancer? Laryngoscope, 115(3), 486-491. doi: 10.1097/01.mlg.0000157851.24272.41

Rees, L. E., Pazmany, L., Gutowska-Owsiak, D., Inman, C. F., Phillips, A., Stokes, C. R., .. . Birchall, M. A. (2008). The mucosal immune response to laryngopharyngeal reflux. Am J Respir Crit Care Med, 177(11), 1187-1193. doi: 10.1164/rccm.200706-895OC

Salvioli, B., Belmonte, G., Stanghellini, V., Baldi, E., Fasano, L., Pacilli, A. M., . . Corinaldesi, R. (2006). Gastro-oesophageal reflux and interstitial lung disease. Dig Liver Dis, 38(12), 879-884. doi: 10.1016/j.dld.2006.05.012

Sung, M. W., Roh, J. L., Park, B. J., Park, S. W., Kwon, T. K., Lee, S. J., \& Kim, K. H. (2003). Bile acid induces cyclo-oxygenase- 2 expression in cultured human pharyngeal cells: a possible mechanism of carcinogenesis in the upper aerodigestive tract by laryngopharyngeal reflux. Laryngoscope, 113(6), 1059-1063. doi: 10.1097/00005537200306000-00027

Sweet, M. P., Patti, M. G., Hoopes, C., Hays, S. R., \& Golden, J. A. (2009). Gastro-oesophageal reflux and aspiration in patients with advanced lung disease. Thorax, 64(2), 167-173. doi: $10.1136 /$ thx.2007.082719

Vaezi, M. F., Qadeer, M. A., Lopez, R., \& Colabianchi, N. (2006). Laryngeal cancer and gastroesophageal reflux disease: a case-control study. Am J Med, 119(9), 768-776. doi: 10.1016/j.amjmed.2006.01.019

Vakil, N. (2010). Disease definition, clinical manifestations, epidemiology and natural history of GERD. Best Pract Res Clin Gastroenterol, 24(6), 759-764. doi: 10.1016/j.bpg.2010.09.009

Vakil, N., van Zanten, S. V., Kahrilas, P., Dent, J., \& Jones, R. (2006). The Montreal definition and classification of gastroesophageal reflux disease: a global evidence-based consensus. Am J Gastroenterol, 101(8), 1900-1920; quiz 1943. doi: 10.1111/j.15720241.2006.00630.x

Vereczkei, A., Horvath, O. P., Varga, G., \& Molnar, T. F. (2008). Gastroesophageal reflux disease and non-small cell lung cancer. Results of a pilot study. Dis Esophagus, 21(5), 457-460. doi: 10.1111/j.1442-2050.2007.00796.x 


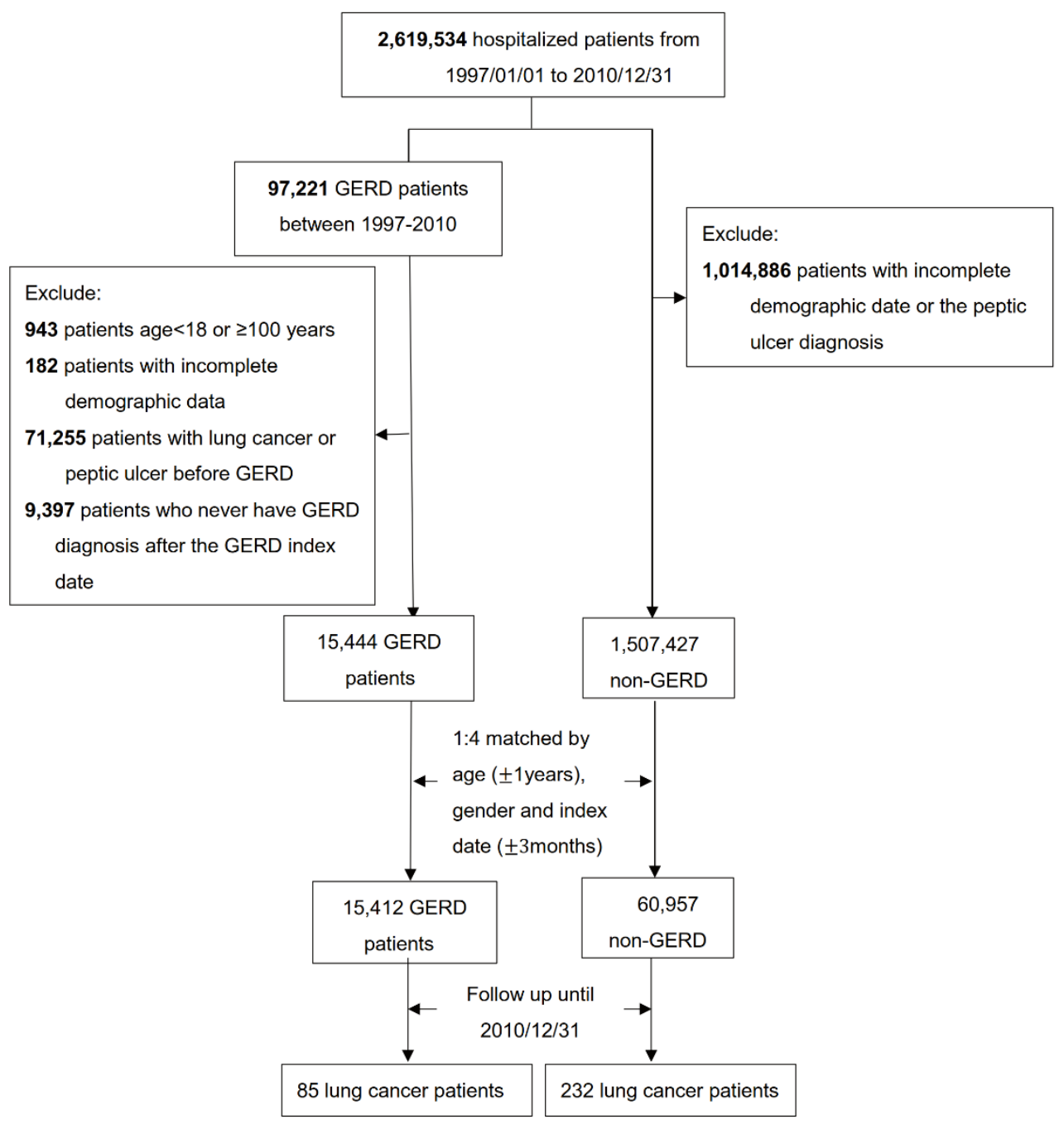

\section{Study algorithm for patient enrollment}


307

308

309

310

311

312

313

314

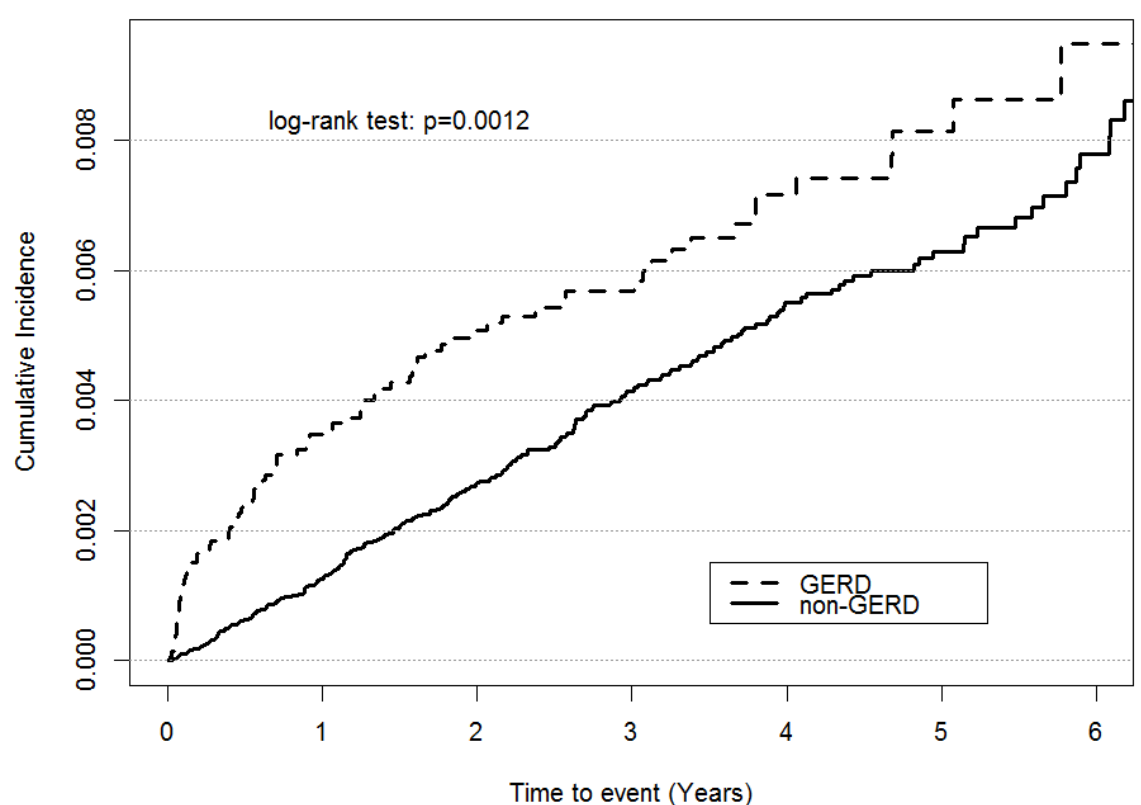

No. of Lung Cancer:

$\begin{array}{rlcccccc}\text { GERD } & 0 & 50 & 67 & 72 & 80 & 83 & 85 \\ \text { non-GERD } & 0 & 69 & 134 & 179 & 209 & 219 & 228\end{array}$

Figure 2.

Cumulative incidence rate of lung cancer for patients with or without GERD 
315 Table 1. Incidence rates of lung cancer events per 10,000 person-year among gastro-esophageal reflux disease (GERD) and non-

316 GERD group.

\begin{tabular}{lcccr}
\hline Groups & Person-year & Number of lung cancer & Rate (per 10,000 person- & year) \\
\hline GERD & 42555.51 & 85 & 0.0020 & $(0.0016,0.0024)$ \\
non-GERD & 175319.72 & 232 & 0.0013 & $(0.0012,0.0015)$ \\
\hline
\end{tabular}

317 


\begin{tabular}{|c|c|c|c|c|}
\hline Variables & $\begin{array}{c}\text { Number (\%) of patients with } \\
\text { GERD } \\
\mathrm{N}=\mathbf{1 5 , 4 1 2}\end{array}$ & $\begin{array}{c}\text { Number }(\%) \text { of patients } \\
\text { without GERD } \\
\mathrm{N}=60,957\end{array}$ & $\chi^{2}(\mathrm{df})$ & p-value \\
\hline Gender & & & $0.326(1)$ & 0.568 \\
\hline Female & 7849 (50.9) & 31201 (51.2) & & \\
\hline Male & 7563 (49.1) & $29756(48.8)$ & & \\
\hline Age group & & & $4.203(2)$ & 0.122 \\
\hline $18-54$ years & $6503(42.2)$ & $26049(42.7)$ & & \\
\hline $54-64$ & $5961(38.7)$ & $23677(38.8)$ & & \\
\hline$\geq 65$ years & $2948(19.1)$ & $11231(18.4)$ & & \\
\hline \multicolumn{5}{|c|}{ Underlying diseases/conditions } \\
\hline Osteoporosis & & & $15.366(1)$ & $<0.001$ \\
\hline No & $15173(98.5)$ & $60250(98.84)$ & & \\
\hline Yes & $239(1.6)$ & $707(1.2)$ & & \\
\hline Diabetes mellitus & & & $16.298(1)$ & $<0.001$ \\
\hline No & $14078(91.3)$ & $55030(90.3)$ & & \\
\hline Yes & $1334(8.7)$ & $5927(9.7)$ & & \\
\hline Tuberculosis & & & $0.930(1)$ & 0.335 \\
\hline No & $15342(99.6)$ & $60714(99.6)$ & & \\
\hline
\end{tabular}


Yes

$70(0.5)$

Asthma

No

Yes

Chronic obstructive pulmonary diseases

No

Yes

Pneumonia

No

Yes

Pneumoconiosis

No

Yes

Bronchiectasis

No

Yes

Depression

No

Yes

$63(0.4)$
$243(0.4)$

95.058(1)

$<0.001$

14881 (96.6)

531 (3.5)

$59673(97.9)$

1284 (2.1)

$259.590(1)$

$<0.001$

58015 (95.2)

$2942(4.8)$

247.106(1)

$<0.001$

14929 (96.9)

483 (3.1)

$60157(98.7)$

$800(1.3)$

$0.031(1)$

0.861

15401 (99.9)

11 (0.1)

60916 (99.9)

41 (0.1)

28.373(1)

$<0.001$

15349 (99.6)

$60847(99.8)$

$110(0.2)$

$37.239(1)$

$<0.001$
$15242(98.9)$

$170(1.1)$
$60570(99.4)$

$387(0.6)$ 
Anxiety

No

Yes

Hypertension

No

Yes

Dyslipidemia

No

Yes

Chronic liver disease

No

Yes

Congestive heart failure

No

Yes

Atrial fibrillation

No

Yes

Myocardial infarction
$365.826(1)$

$<0.001$

58337 (95.7)

$2620(4.3)$

9.954(1)

0.002

12031 (78.1)

48291 (79.2)

3381 (22.0)

$12666(20.8)$

11.772(1)

$<0.001$

13993 (90.8)

1419 (9.2)

55871 (91.7)

$5086(8.3)$

75.039(1)

$<0.001$

14865 (96.5)

547 (3.6)

59546 (97.7)

$1411(2.3)$

21.013(1)

$<0.001$

15118 (98.1)

294 (2.0)

60101 (98.6)

856 (1.4)

4.635(1)

0.031

15274 (99.1)

$138(0.9)$
$60514(99.3)$

$443(0.7)$ 


\begin{tabular}{|c|c|c|c|c|}
\hline No & $15316(99.4)$ & $60600(99.4)$ & & \\
\hline Yes & $96(0.62)$ & $357(0.6)$ & & \\
\hline Stroke & & & 19.921(1) & $<0.001$ \\
\hline No & $14581(94.6)$ & $58190(95.5)$ & & \\
\hline Yes & $831(5.4)$ & $2767(4.6)$ & & \\
\hline Peripheral vascular disease & & & $0.720(1)$ & 0.720 \\
\hline No & $15350(99.6)$ & $60724(99.6)$ & & \\
\hline Yes & $62(0.4)$ & $233(0.7)$ & & \\
\hline Chronic kidney diseases & & & $32.318(1)$ & $<0.001$ \\
\hline No & $15083(97.9)$ & $60050(98.5)$ & & \\
\hline Yes & $329(2.1)$ & $907(1.5)$ & & \\
\hline Coronary artery diseases & & & $32.782(1)$ & $<0.001$ \\
\hline No & $15051(97.7)$ & $59947(98.3)$ & & \\
\hline Yes & $361(2.3)$ & $1010(1.7)$ & & \\
\hline
\end{tabular}


Table 3. Crude hazard ratios (HR) among gastro-esophageal reflux disease (GERD) and non-GERD group.

\begin{tabular}{lccc}
\hline Variables & beta value & $\begin{array}{c}\text { crude HR } \\
(95 \% \mathrm{Cl})\end{array}$ & p value \\
\hline GERD & 0.43 & $1.53(1.19-1.98)$ & 0.001 \\
Osteoporosis & 0.59 & $1.80(0.88-3.69)$ & 0.110 \\
Diabetes mellitus & 0.05 & $1.05(0.76-1.46)$ & 0.752 \\
Asthma & 0.20 & $1.22(0.65-2.29)$ & 0.538 \\
Chronic obstructive pulmonary diseases & 0.30 & $1.34(0.92-1.96)$ & 0.125 \\
Pneumonia & 0.40 & $1.49(0.76-2.90)$ & 0.245 \\
Bronchiectasis & 0.69 & $2.00(0.18-22.06)$ & 0.571 \\
Depression & 0.84 & $2.31(0.55-9.69)$ & 0.251 \\
Anxiety & -0.08 & $0.92(0.53-1.60)$ & 0.765 \\
Hypertension & -0.11 & $0.90(0.69-1.17)$ & 0.429 \\
Dyslipidemia & -0.02 & $0.98(0.65-1.46)$ & 0.913 \\
Chronic liver disease & -0.25 & $0.78(0.36-1.71)$ & 0.539 \\
Congestive heart failure & -0.88 & $0.42(0.15-1.19)$ & 0.102 \\
Atrial fibrillation & -1.23 & $0.29(0.07-1.25)$ & 0.098 \\
Stroke & -0.42 & $0.66(0.41-1.05)$ & 0.079 \\
Chronic kidney diseases & -0.31 & $0.73(0.33-1.60)$ & 0.433 \\
Coronary artery diseases & -0.28 & $0.76(0.36-1.58)$ & 0.458 \\
\hline
\end{tabular}

